\title{
Off-body Wireless Link Simulation Framework Using Deterministic Channel Modeling
}

\author{
Luigi Vallozzi ${ }^{1}$, Hendrik Rogier ${ }^{1}$, \\ ${ }^{1}$ Electromagnetics group, Department of Information Technology, Ghent University, Ghent, Belgium \\ Luigi.Vallozzi@UGent.be, Hendrik.Rogier@UGent.be
}

\begin{abstract}
Body-centric communication concerns wireless links in which the human body represents the central element, exchanging data by means of wearable antennas with a different node in the external environment or on the human body itself. During the link design phase, it is very advantageous to be able to model the link and predict its performance before its real implementation, resulting in reduction of design time and costs. In the present contribution, we propose a novel computer simulation framework to describe a complete offbody communication link between a human user and a remote base station, including all the components of the communication link such as wearable and fixed antennas, human body and propagation channel at the physical level, also taking into account the digital data transmission at link level. The proposed framework was constructed by applying the most appropriate modeling tools for each component, including ray tracing for the deterministic channel modeling. The framework provides as output the most common link performance parameters such as Bit-Error-Rate (BER), received Signal-to-Noise Ratio (SNR) and channel correlations. The validity of the proposed method was verified by comparing the figures of merit of a real off-body communication link, studied during a previous measurement campaign, with the ones obtained by the proposed simulation framework. A good agreement is obtained in terms of both BERs and SNRs.
\end{abstract}

Index Terms-Body-centric communication, off-body communication, ray tracing.

\section{INTRODUCTION}

Body-centric communication has been object of intense research in the last decade [1]. Wireless communication links, where the human body represents one or more communication nodes, have numerous applications such as, for example, monitoring of life signs and activity of rescue workers or hospital patients.

One particular class of body-centric communication is known as off-body communication: it takes place between a human user and an external node, which can be a remote fixed base station or another moving human user located at relatively short distance. This paper focuses on the modeling of an off-body communication link that, at the physical level, is composed of several constituting parts, in particular wearable antennas, human body and wireless propagation channel.

In order to assess the performance of an off-body communication link, obviously the most accurate method is to perform experimental tests on the real system. Unfortunately, a measurement campaign on a real off-body communication system can only be performed after the full implementation and, in addition, it can be time consuming and thus expensive. Therefore, in order to reduce design time and costs, the availability of simulation tools, capable of predicting the link performance before its implementation, it's very important. The aim of the present work is to construct such a simulation framework for the performance prediction of the complete off-body communication link, in narrow-band conditions. In currently available literature on the subject, few work has been performed with such an aim, mainly focusing on the propagation channel, rather than the complete communication link, without considering global performance parameters such as BER or SNR [2], [3]. Although research has concentrated on the modeling of the individual link components, resulting in a large variety of software tools for individual parts such as antennas and communication channel, few attention was addressed to the development of a single comprehensive tool to model the complete wireless link in terms of performance parameters such as BER, SNR and channel correlations. Therefore, the proposed integrated framework models the antennas, the human body, the wireless channel and the digital data transmission through the communication link.

The proposed simulation framework, described in Section II, applies several tools to model the different constituting parts of the off-body link, being a 3D full-wave EM solver to model wearable antennas on the human body, and a ray tracer that describes the multipath propagation channel in a deterministic way. Moreover, at link level, the proposed framework simulates the transmission of pseudo-random digital data to calculate BER, SNRs and channel correlations.

For verification purposes, the proposed method was applied in Section III to reproduce a multi-antenna off-body communication system that was previously realized [4], and a good agreement was obtained between the performance parameters computed by means of the proposed method and the ones obtained by measurements on the real system.

\section{DESCRIPTION OF THE MODELING FRAMEWORK}

A Multiple-Input Multiple-Output (MIMO) off-body communication system consists of a link between a multi-antenna system, worn on a human body, and a remote base station equipped with multiple antennas, between which a wireless propagation channel is present. A conceptual scheme of such a type of communication link is depicted in Fig. 1. The proposed framework consists of two parts, the first representing the 


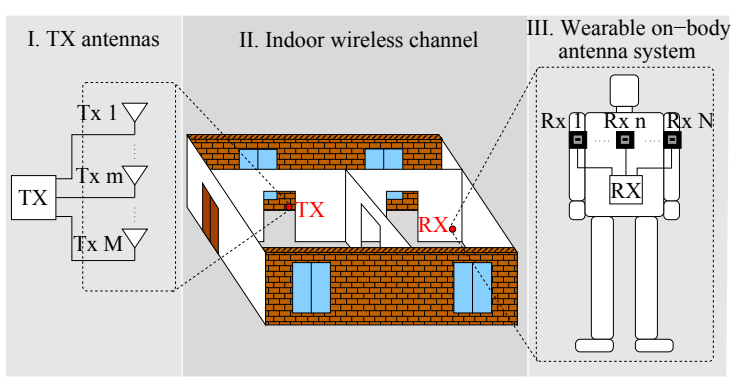

Fig. 1. Scheme of an off-body indoor wireless communication system

off-body link at the physical-level, i.e. the physical channel between the transmit (TX) and receive (RX) antennas, and the second one reproducing the link-level, representing the transmission and processing of information through the link. We now discuss both tools in more detail.

\section{A. Physical-level model: TX-RX channel response}

1) Modeling tools: The proposed modeling framework combines the following software tools, to describe the complete transmission chain at the physical level:

- Full-wave 3D EM solver, modeling the wearable on-body antennas, as well as the base station antennas, including wearable antennas and a voxel-based human body model. Given the geometrical and dielectric description of the entire structure, for the TX and RX sides, we calculate the $3 \mathrm{D}$ radiation vector pattern $\bar{F}(\theta, \phi)$ as a function of the observation angles, being the elevation angle $\theta$ and the azimuth angle $\phi$.

- Ray-tracer, computing the electromagnetic field in a specified portion of the simulation space, given the geometrical and dielectric description of the propagation environment (e.g. an indoor office environment), as well as the description of the source of the EM-field (i.e. TX antenna(s)) in terms of radiation pattern, transmitted power and position. The ray tracer evaluates the EM field in a fully deterministic way, following the laws of the geometrical theory of diffraction. This high-frequency asymptotic technique, firstly applied to radiowave propagation in [5], traces the EM waves as plane waves following rectilinear paths through the environment, undergoing transmission, reflection and diffraction, as displayed in Fig. 2. The ray tracer provides as an output two vectorial channel responses for horizontal and vertical polarization $\left(\bar{g}^{V}, \bar{g}^{H}\right)$, representing the total electric field produced at a given position, by a horizontally and vertically polarized isotropic radiator, respectively.

The EM field distribution can be computed by analytical, empirical, stochastical and deterministic techniques. We chose to use the last method since it provides an exact computation of the particular propagation channel under study, in contrast to the other methods that describe an average channel environment with given general characteristics. Deterministic methods, such as ray tracing, are thus exact and more flexible, but computationally

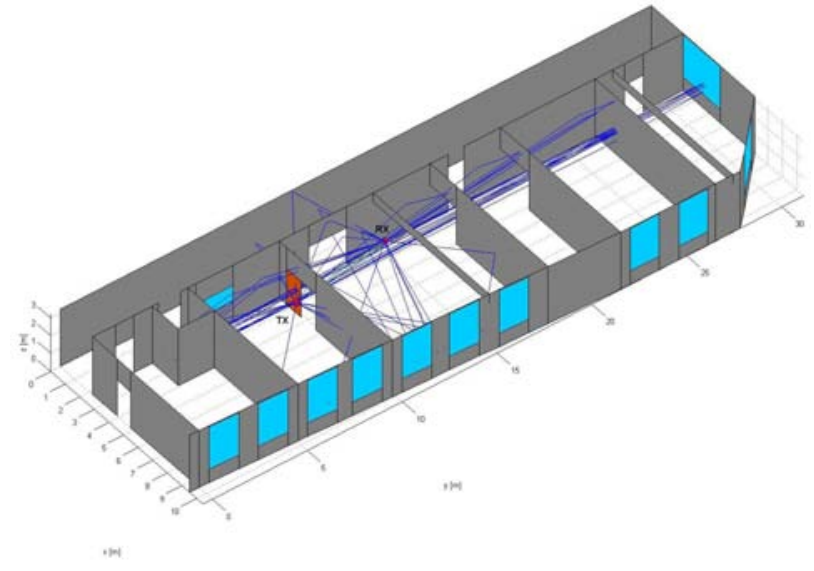

Fig. 2. Propagation rays in a multipath indoor environment computed by the ray tracer program

more expensive than other methods. Yet, by applying acceleration techniques, the computational burden can be significantly reduced, making this technique more appealing than in the past.

The total TX-RX narrow-band scalar channel response, for a given TX and RX antenna at given spatial positions, is obtained by combining the antenna radiation vector pattern computed by the 3D EM solver, and the vectorial channel responses calculated by the ray tracer, illustrated as follows.

2) Meaning of scalar channel response: let us consider the communication link between one particular couple of TX$\mathrm{RX}$ antennas, placed at fixed positions in the propagation environment space. As shown in Fig. 3, a scalar propagation channel response can be defined between the TX and RX antenna ports. This is indicated by $h=|h| e^{j \phi_{h}}$ and represents the ratio between the output signal at the RX antenna port, over the input signal at the TX antenna port, i.e. $h=v_{r x} / v_{t x}$.

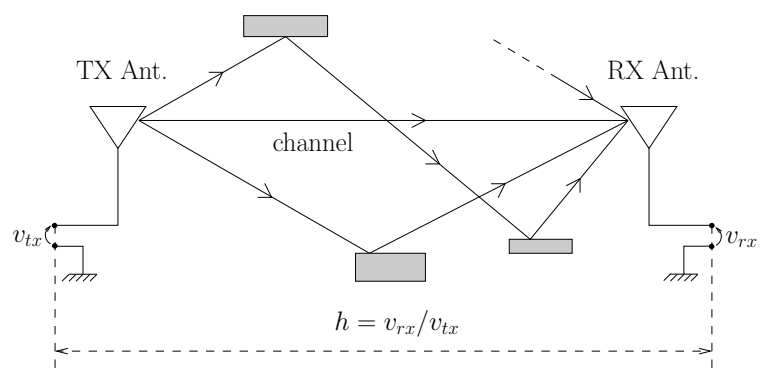

Fig. 3. Scalar channel response between the terminals of TX and RX antennas

The scalar channel response represents the narrowband, static channel response between the terminals of the transmitting and receiving antennas. In the following it will be explained how the scalar channel response is calculated in the proposed framework, by combining the outputs of the ray tracer and the 3D full-wave EM solver.

The ray tracer provides, as an output, the two so-called vectorial channel responses $\bar{g}^{(V)}, \bar{g}^{(H)}$, representing the components of the electric field, at a given receiver antenna 
position, for vertically and horizontally oriented isotropic transmit antennas, respectively, which are calculated by the ray tracer as superposition of $L$ rays. In particular, the $l^{t h}$-ray component of the vectorial channel response can be written in matrix notation, as the product of a tensor, representing the channel response for the $l^{t h}$ ray, and the radiation vector of the isotropic antenna:

$$
\bar{g}_{l}^{(p o l)}=\overline{\bar{G}}_{l} \cdot \bar{F}_{i s o, l}^{(p o l)}, \quad \text { pol }=\{H, V\}
$$

where the tensor $\overline{\bar{G}}_{l}$, computed by the ray tracer, is independent from the orientation of the considered isotropic transmit antenna ( $p o l)$ and depends on the reflections, transmissions, diffractions as well as on the propagation factor experienced by the $l^{\text {th }}$ ray. The scalar channel response $h$ can be obtained from $\bar{g}_{l}^{(H)}$ and $\bar{g}_{l}^{(V)}(l=1, \ldots, L)$, by means of an appropriate weighted sum of the $L$ components of the two responses, with proper coefficients calculated on the basis of the radiationvector pattern of the actual transmit antenna, and after inclusion of the receive antenna radiation-vector pattern.

3) Inclusion of the TX antenna radiation pattern: the TX antenna is completely described by its $3 \mathrm{D}$ radiation-vector pattern, $\bar{F}_{t x}(\theta, \phi)=F_{x, t x}(\theta, \phi) \hat{x}+F_{y, t x}(\theta, \phi) \hat{y}+F_{z, t x}(\theta, \phi) \hat{z}$, obtained from the $3 \mathrm{D}$ full-wave solver. In order to include its effect in the channel responses, weighting factors must be applied to each ray component of the two channel responses, $\bar{g}_{l}^{(H)}, \bar{g}_{l}^{(V)}, l=1, \ldots, L$, each one associated with the direction of departure of the $l^{t h}$ ray, $\left(\theta_{D, l}, \phi_{D, l}\right)$. Such a procedure is performed for each ray $l=1, . ., L$, as follows.

The radiation vector of the actual TX antenna, for the $l^{\text {th }}$ ray (i.e. for the angle of departure $\left(\theta_{D, l}, \phi_{D, l}\right)$ ), can always be written as a linear combination of the radiation vectors of the horizontal and vertical isotropic antennas, i.e. :

$$
\bar{F}_{t x, l}=C_{l}^{(H)} \bar{F}_{i s o, l}^{(H)}+C_{l}^{(V)} \bar{F}_{i s o, l}^{(V)}
$$

where $C_{l}^{(H)}, C_{l}^{(V)}$ represent the desired weighting factors for the $l^{\text {th }}$ ray, for the horizontal and vertical vectorial channel responses, respectively. They are easily calculated since $\bar{F}_{t x, l}$, $\bar{F}_{i s o, l}^{(H)}$, and $\bar{F}_{i s o, l}$ are known quantities. The field components of the actual received field, i.e. accounting for the actual TX antenna, for the $l^{t h}$ ray and for the two cases $p o l=\{H, V\}$, are then obtained by multiplying the vectorial channel responses for the corresponding weighting factors, i.e. :

$$
\left\{\begin{array}{c}
\bar{E}_{r x, l}^{(H)}=C_{l}^{(H)} \bar{g}_{l}^{(H)}=C_{l}^{(H)} \overline{\bar{G}}_{l} \cdot \bar{F}_{i s o, l}^{(H)} \\
\bar{E}_{r x, l}^{(V)}=C_{l}^{(V)} \bar{g}_{l}^{(V)}=C_{l}^{(V)} \overline{\bar{G}}_{l} \cdot \bar{F}_{i s o, l}^{(V)}
\end{array}\right.
$$

Finally, the total electric field for each $l^{\text {th }}$ ray, accounting the actual TX antenna radiation pattern, is obtained by superposition of the two components for the $H$ and $V$ cases:

$$
\bar{E}_{r x, l}=\bar{E}_{r x, l}^{(H)}+\bar{E}_{r x, l}^{(V)}=\overline{\bar{G}}_{l} \cdot \bar{F}_{t x, l}
$$

4) Inclusion of the $R X$ antenna radiation pattern: once the actual field impinging on the receiver antenna is known, for each of the $L$ rays, it is straightforward to include the effect of the receive antenna radiation pattern, obtaining the scalar channel $h=|h| e^{j \varphi(h)}$, between the TX and RX antenna terminals.

It is well known that the voltage at the receiving antenna terminals is related to the received field and to the RX antenna radiation vector by means of the law [6]:

$$
v_{r x}=\alpha \bar{E}_{r x} \cdot \bar{F}_{r x}
$$

where $\bar{F}_{r x}$ is the receive antenna radiation vector, for a unit current $I_{t}=1 \mathrm{~A}$ at its terminals, and:

$$
\alpha=-\frac{2 j \lambda}{Z_{c}}
$$

The inclusion of the RX antenna pattern, and the determination of the scalar channel response $h$ proceeds as follows: from the knowledge of the the 3D radiation pattern of the RX antenna (obtained by full-wave simulations) and the angles of arrival of each $l^{t h}$ ray, $\left(\theta_{A, l}, \phi_{A, l}\right), l=1, \ldots, L$, computed by ray tracing, the voltage at the RX antenna terminals $v_{r x}$ is obtained as the superposition of the contributions of $L$ rays, weighted by the RX-antenna radiation vector along the directions of arrival:

$$
v_{r x}=\alpha \sum_{l=1}^{L} \bar{E}_{r x}\left(\theta_{A, l}, \phi_{A, l}\right) \cdot \bar{F}_{r x}\left(\theta_{A, l}, \phi_{A, l}\right)
$$

where $\left(\theta_{A, l}, \phi_{A, l}\right)$ indicates the direction of arrival of the $l^{\text {th }}$ ray. Then, the scalar channel response $h$ is simply obtained as:

$$
h=\frac{v_{r x}}{v_{t x}}
$$

It is important to remark that the scalar channel response $h$ expresses the narrow-band, static channel response, referred to given positions of the transmitter and receiver. Actually, for the performed simulations, the scalar channel calculation was repeated for several positions of the mobile receiver. Thus, if the total number of receiver points is $N$, the scalar channel response, over all points, can be expressed as $h(k), k=1, \ldots, N$. Moreover, the scalar channel calculation was repeated, in general, for a given number of antennas or antenna ports at the receiving side, denoted by $n_{r}$. Thus we obtain, in general, $n_{r}$ scalar channel responses, indicated as $h_{j}(k), j=1, \ldots, n_{r}, k=1, \ldots, N$. These are then used to compute the link performance parameters by means of Monte Carlo simulation to generate a digital data transmission.

\section{B. Link-level model: digital data transmission simulation}

In general, for an off-body communication system with $n_{t}$ TX antennas (base station) and $n_{r}$ on-body RX antennas, $n_{r} \times n_{t}$ channel responses are calculated, over a specified space of $N$ points, resulting in a MIMO system. In our study we decided to focus on the particular case of off-body links with a single TX antenna and multiple on-body RX antennas, resulting in $n_{r}$ channel responses computed over a spatial grid of $N$ points, i.e. $h_{i}(k), i=1, \ldots, n_{r}, k=1, \ldots, N$. The knowledge of these channel responses provides the necessary information to simulate the performance of the complete communication system at the link level, that is, when a digital 
data transmission is performed between TX and RX. A computer routine was implemented to perform such a simulation, including modulation/demodulation, diversity combining and signal detection at the RX side, generating as a result the link performance parameters such as BER, SNRs, channel correlations and statistics. A scheme of the constructed routine, is displayed in Fig 4.

In the base-band communication link simulation, first a sequence of pseudo-random bits is generated, mapped into a symbols stream, $c(k)$, and then transmitted across the communication channels, with the computed channel gains $h_{1}(k), \ldots, h_{n_{r}}(k)(k=1, \ldots, N)$. At the RX side, consisting of $n_{r}$ branches, Additive White Gaussian Noise (AWGN) with power spectral density $N_{0, i}$, is added to the received signals. The noisy $n_{r}$ received signals can be written as:

$$
r_{i}(k)=c(k) h_{i}(k)+n_{i}(k), \quad i=1, \ldots, n_{r}
$$

Then, the received signals undergo combining which, in our particular case, is Maximal Ratio Combining (MRC). In order not to complicate the discussion, we assume perfect channel gain knowledge at the receiver. In particular, the signal obtained after MRC can be written as:

$$
d(k)=\sum_{i=1}^{n_{r}} r_{i}(k) \frac{h_{i}^{*}(k)}{N_{0, i}}
$$

A decision, $\hat{c}_{k}$, is then taken based on $d(k)$ according to the maximum likelihood criterium. If $L_{D}$ is the the total number of simulated bits, the BER value is calculated as $B E R=N_{e r r} / L_{D}$, where $N_{\text {err }}$ is the total number of bit (or equivalently symbol) errors.

The estimation of the average BER, in a certain receiver space composed of $N$ points, is carried out by simulating a transmission of $L_{D}$ bits (typically $L_{D}=10^{5}$ or $10^{6}$ ), for each of the $N$ scalar channel response points $h_{i}(k), k=1, \ldots, N$, $i=1, \ldots, n_{r}$, and for a given number of $E_{b} / N_{0}$ levels (with $N_{0}$ being the average of the $\left.N_{0, i}, i=1, \ldots, n_{r}\right) . N$ AWGN $\operatorname{BER}_{i}\left(E_{b} / N_{0}\right)$ curves are thus obtained, one for each of the $N$ points composing the receiver space. After that, the average $\operatorname{BER}\left(E_{b} / N_{0}\right)$ curve on the considered receiver space can be calculated as the average of the above mentioned $N$ AWGN $\operatorname{BER}_{i}\left(E_{b} / N_{0}\right)$ curves.

\section{CASE STUdy AND RESUlts}

In this section, a case study is described and the obtained results discussed. The study is limited to receive diversity schemes only, i.e with a single antenna at the fixed transmit side and multiple on-body antennas with multiple polarizations at the receive side. First, the considered propagation scenario is described. Next, the obtained results are presented and commented.

\section{A. Description of the modeled scenario}

The modeled propagation environment consists of an indoor office environment in which the measurement campaign described in [4] was performed. A model of this environment was built including walls, door apertures, windows, floor
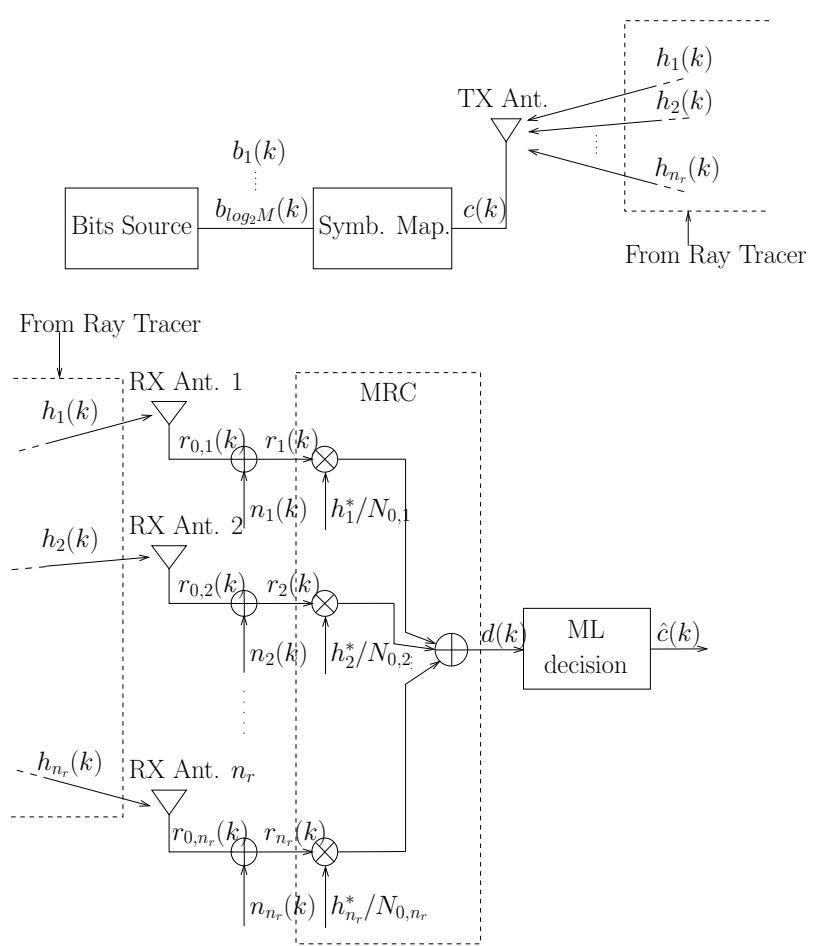

Fig. 4. Scheme of the base-band digital data transmission simulation implemented in the simulation framework

and ceiling, as well as additional furniture elements such as desks, metal cupboards, heating devices and metallic PC cases. First, a CAD-drawing of the environment was made, with the geometrical description of each object and their dielectric parameters $\varepsilon_{r}$ and $\sigma$. After that, such a model, in an appropriate format, was given as input to the ray tracer, in which the receiver space can be specified, that is the area of the propagation environment for which the ray tracer calculates the EM fields. A rectilinear path was chosen as receiver space for the considered case study. The considered rectilinear grid is a rectangular area, in which the resolution $r$ is set equal to the side of the area, as depicted in Fig. 5, oriented along a given direction. Such a receiver space was used to simulate a received field distribution over a length along which both small-scale fading and large-scale fading are observed, since the length of the path $D$ was set much larger than $10 \lambda$. In our particular case, we chose a resolution $r=2 \mathrm{~cm}$ and a path length $D=5.9 \mathrm{~m}$, corresponding to about $48 \lambda$ and $N=295$ calculation points.

B. Simulations of a $1 \times 4$ off-body link between base station and realistic on-body wearable antennas on human body

The simulations presented in this section aim to reproduce the measurement campaign shown in [4]. For this reason, the rectilinear path chosen as receiver space, has the same length and location of the path followed by the test person in the above mentioned measurement campaign.

Two dual polarized patch antennas are considered, worn on front and back side of a human body, whose radiation vector 


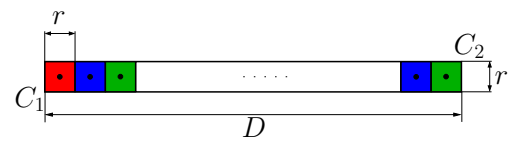

Fig. 5. Rectilinear-path receiver space used in the simulation

patterns are obtained by 3D full-wave simulations performed by CST Microwave Studio, considering only the upper portion of the human body, which has a larger influence on the gain patterns of the antennas.

First, the two $1 \times 2$ off-body sub-links composing the total system, each corresponding to one dual-polarized antenna, either deployed at the front or at the back side of the human users, are simulated, followed by the simulation of the total $1 \times 4$ link. Next, their performances are compared. In Fig. 6 the simulated $\operatorname{BER}\left(E_{b} / N_{0}\right)$ resulting from the simulation of the $1 \times 2$ system with a DPPA at the front is shown. One can see that the simulated performance is worse than the one for two Rayleigh uncorrelated channels, having the same powers as for the simulated channels, being $P_{r x 1}=7.67 \cdot 10^{-9} \mathrm{~W}$ and $P_{r x 1}=5.35 \cdot 10^{-9} \mathrm{~W}$. The BER matches very well the one for two uncorrelated Nakagami channels with parameters obtained by fitting, resulting in $\mu_{1, \text { fit }}=0.5, \Omega_{1, \text { fit }}=5.59 \cdot 10^{-9} \mathrm{~W}$ for channel 1 , and $\mu_{2, \text { fit }}=0.5, \Omega_{2, \text { fit }}=4.72 \cdot 10^{-9} \mathrm{~W}$ for channel 2. The simulated channels power correlation is equal to $\rho_{\text {pow }}=0.50$, calculated using a moving average window with a width equal to $1.3[\mathrm{~m}]$, that corresponds to $N=65$ receiver points and a length that is slightly larger than $10 \lambda$.

In Fig. 7 the simulated BER curve of the total $1 \times 4$ system using front and back DPPAs is displayed, and compared with the $1 \times 2$ case. One notices that the performance of the $4^{\text {th }}$-order diversity system shows a considerable improvement with respect to the $1 \times 2$ case, in good agreement with the measurement campaign results described in [4].

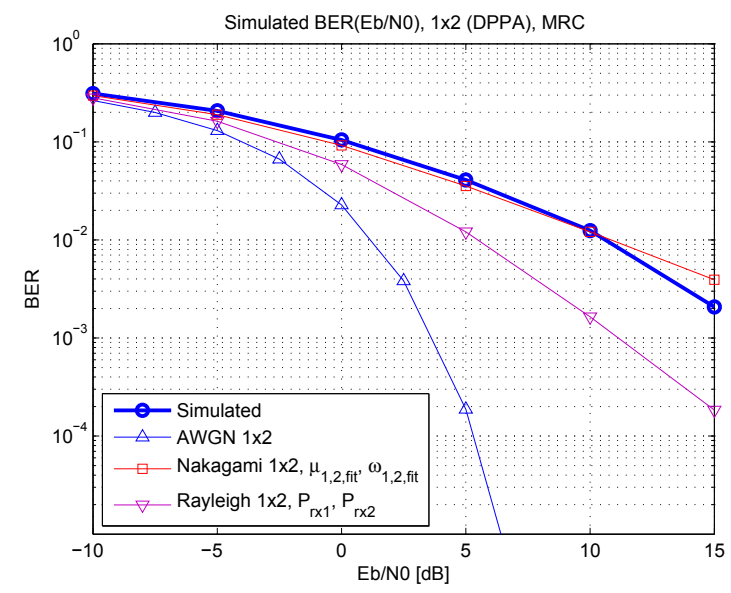

Fig. 6. Simulated bit error rate curve, together with theoretical and fitted BER curves of known distributions, for the $1 \times 2$ link, with one RX dual polarized textile patch antenna, worn at the front of the human body

Most importantly, a good agreement in terms of BER was

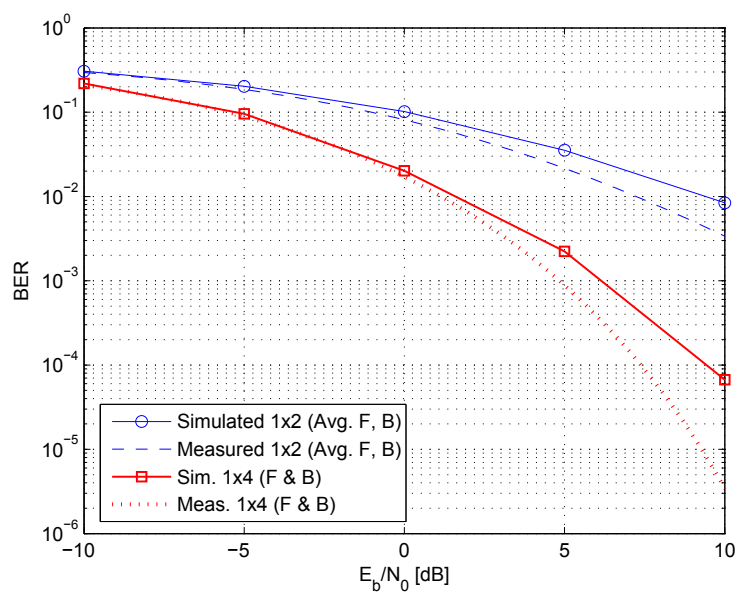

Fig. 7. Comparison between the measured BER curves of a 1x4 off-body communication system and the simulated ones obtained by the proposed simulation framework

observed between the measured performance and the predicted link quality obtained by the proposed framework. Fig. 7 shows a good match between measurement and simulation, both for the $1 \times 4$ off-body link and for the two $1 \times 2$ links.

\section{CONCLUSION}

A novel and effective modeling framework for the entire off-body communication link was developed by combining different tools for the different composing units of the communication channel. The proposed framework, which relies on deterministic channel modeling, was tested by modeling a $4^{\text {th }}$-order diversity off-body communication link between a fixed base station and a human-body equipped with two dualpolarized patch antennas, and simulating its performance in terms of BER, yielding results that are in sufficiently good agreement with the ones obtained by measurements on the real system, proving the effectiveness of the proposed technique. Future work will aim to extend the current model, valid for the narrowband case, to wideband applications.

\section{REFERENCES}

[1] P. S. Hall and Y. Hao, Antennas and Propagation for Body-Centric Wireless Communications. Boston/London: Artech House, 2006.

[2] S. Chamaani, Y. Nechayev, P. Hall, and S. Mirtaheri, "In-body to off-body channel modelling," in Antennas and Propagation Conference (LAPC), 2010 Loughborough, 2010, pp. 609-612.

[3] M. Sarestuniemi, T. Tuovinen, M. Hamalainen, and J. Iinatti, "Finite integration technique for modelling of wban communication links in complex environments," in Medical Information and Communication Technology (ISMICT), 2013 7th International Symposium on, 2013, pp. $154-158$.

[4] L. Vallozzi, P. Van Torre, C. Hertleer, H. Rogier, M. Moeneclaey, and J. Verhaevert, "Wireless Communication for Firefighters Using DualPolarized Textile Antennas Integrated in Their Garment," IEEE Trans. Antennas Propag., vol. 58, no. 4, pp. 1357-1368, Apr. 2010.

[5] J. McKown and J. Hamilton, R.L., "Ray tracing as a design tool for radio networks," Network, IEEE, vol. 5, no. 6, pp. 27-30, Nov. 1991.

[6] C. A. Balanis, Antenna Theory: Analysis and Design, 2nd ed. New York: John Wiley \& Sons, 1997. 